\title{
A 2-Year-Old Boy With Refractory Kawasaki Disease Who Developed a Giant Aneurysm After Successful Treatment With Infliximab
}

\author{
Saki Endo a, c, Masato Kimura, c, d, Hisao Yaoita ${ }^{\mathrm{a}}$, Chiharu Ota ${ }^{\mathrm{a}}$, \\ Ryouichi Oonuma ${ }^{\mathrm{b}}$, Setsuko Kitaoka ${ }^{\mathrm{b}}$
}

\begin{abstract}
Kawasaki disease (KD) is the leading cause of systemic vasculitis syndrome in infants and in young children, although its cause has still not been elucidated. Coronary aneurysm $(\mathrm{CA})$ is a major and important complication of KD. Patients resistant to intravenous immunoglobulin (IVIG) treatment are at a high risk of CA. Infliximab (IFX) is a chimeric monoclonal antibody that specifically binds to tumor necrosis factor-alpha (TNF- $\alpha$ ) and has been successfully used in some cases of IVIG-refractory KD. However, optimal timing for the administration of IFX has not yet been determined. We present a case of a 2-year-old boy with IVIG-refractory KD who developed a giant right coronary artery (RCA) aneurysm after successful treatment with IFX. Treatment with IVIG (2 g/kg), prednisolone (PSL, $2 \mathrm{mg} / \mathrm{kg} /$ day), and aspirin $(50 \mathrm{mg} / \mathrm{kg} /$ day) was initiated on day 2 because of the fulfillment of KD criteria. Additional IVIG ( $2 \mathrm{~g} / \mathrm{kg})$ and ulinastatin $(20,000$ $\mathrm{U} / \mathrm{kg}$ ) treatment on day 5 and intravenous administration of IFX (5 $\mathrm{mg} / \mathrm{kg}$ ) on day 9 effectively lowered the patient's fever and improved laboratory data. However, the RCA continued to balloon into a giant $\mathrm{CA}$, from $7.3 \mathrm{~mm}$ on day 9 to $14.3 \mathrm{~mm}$ in diameter at 2 months. IFX is one treatment option for IVIG-refractory KD. However, the timing and indication of IFX treatment should be carefully determined.
\end{abstract}

Keywords: Giant coronary aneurysm; Infliximab; IVIG refractory; Kawasaki disease; TGF- $\beta$; Thrombus; TNF- $\alpha$

\section{Introduction}

Kawasaki disease (KD) is defined as acute systemic vasculitis

Manuscript submitted August 15, 2019, accepted August 28, 2019

aDepartment of Pediatrics, Tohoku University School of Medicine, Miyagi 980-8574, Japan

bepartment of Pediatrics, Sendai Medical Center, Sendai, Miyagi, Japan ${ }^{\mathrm{c}}$ The two authors contributed equally as the first authors of the manuscript. ${ }^{\mathrm{d} C}$ Corresponding Author: Masato Kimura, Department of Pediatrics, Tohoku University Graduate School of Medicine, 1-1 Seiryocho, Aoba-ku, Sendai, Miyagi 980-8574, Japan. Email: mkimura774@med.tohoku.ac.jp

doi: https://doi.org/10.14740/ijcp345 in infants and young children with six clinical symptoms: persistent high fever, bilateral conjunctival congestion, rash, lip redness, nonpurulent cervical lymphadenopathy, and indurative edema of the extremities. However, the underlying cause of $\mathrm{KD}$ remains unknown [1]. Coronary aneurysm (CA) is a major complication of $\mathrm{KD}$. Thus, preventing the formation of a CA, especially a giant CA, is a major and important objective in the treatment in acute-phase KD. The incidence of a giant $\mathrm{CA}$, defined as a diameter larger than $8 \mathrm{~mm}$, is rare in Japan $(0.22-0.40 \%)$ [2]. However, patients with a giant CA are at high risk for myocardial infarction and/or cardiac death due to progressive coronary artery calcification and thrombus formation in the long term. At present, intravenous immunoglobulin (IVIG, $2 \mathrm{~g} / \mathrm{kg}$ ) and an anti-inflammatory dose of aspirin (30 $50 \mathrm{mg} / \mathrm{kg}$ ) is the worldwide standard for first-line therapy. The rate of resistance to first-time IVIG treatment (refractory KD) is approximately $10-20 \%$, so patients are at risk of CA formation. IVIG, prednisolone (PSL), ulinastatin, cyclosporine, and plasma exchange have been reported as effective additional treatment choices for refractory KD. Infliximab (IFX), a chimeric monoclonal antibody that specifically binds to tumor necrosis factor-alpha (TNF- $\alpha$ ), has been successfully used in some cases of IVIG-refractory KD. Many case series and randomized controlled studies have revealed that IFX is effective and well-tolerated in patients with IVIG-refractory KD [3-7]. However, the optimal timing and indications of IFX have not been fully elucidated.

IFX is effective and safe for refractory $\mathrm{KD}$, especially for patients without CA before IFX. However, for patients with CA before IFX administration, the timing and indication of IFX treatment should be carefully determined.

\section{Case Report}

A 2-year-old boy was referred to our university hospital due to an aneurysm in his right coronary artery (RCA) that gradually dilated after two rounds of intravenous IVIG and PSL therapy. He had no past medical history and his two older sisters and his parents had no past histories of KD, connective tissue disease, or autoimmune disease. He had received all vaccinations as scheduled in Japan Pediatric Society. Three days before acquiring a fever, his family noticed redness of his eyes without discharge. After 2 days of a high 
Table 1. Laboratory Data

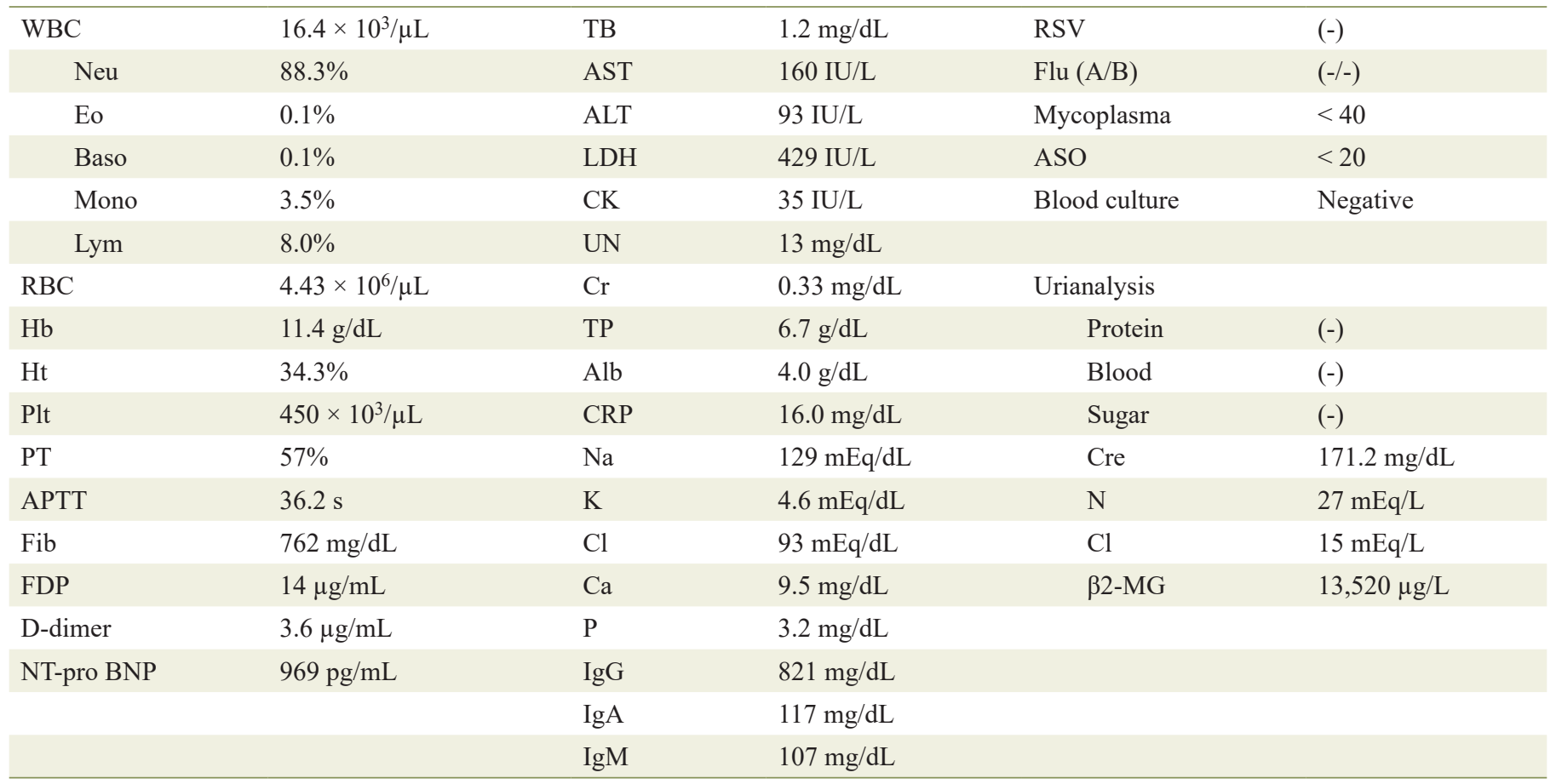

Alb: albumin; ALT: alanine aminotransferase; APTT: activated partial thromboplastin time; AST: aspartate aminotransferase; CK: creatine kinase; Cr: creatinine; CRP: C-reactive protein; FDP: fibrin and fibrinogen degradation product; Hb: hemoglobin; Ht: hematocrit; LDH: lactate dehydrogenase; NT-pro BNP: N-terminal pro brain natriuretic peptide; PIt: platelet; PT: prothrombin time; RBC: red blood cell; TB: total bilirubin; TP: total protein; UN: urea nitrogen; WBC: white blood cell; RSV: respiratory syncytial virus; ASO: antistreptolysin O.

fever, he was admitted to another hospital and diagnosed with KD. On admission, his weight was $15.0 \mathrm{~kg}(50-75$ th percentile) and his height was $93.5 \mathrm{~cm}(90$ - 97th percentile). His temperature was $39.0^{\circ} \mathrm{C}$ and the rest of vital signs were the following: pulse was 158 beats/min, respiratory rate was 40 cycles/min and blood pressure was 105/65 mm Hg. On physical exam, he was conscious, with irritability, no pallor, no cyanosis, clear ears, regular heart sounds with no audible murmur, good bilateral air entry, soft non-tender abdomen, and no focal deficit with negative meningeal signs. He had bilateral nonexudative conjunctivitis, cervical lymph node enlargement, red lips, a rash on his trunk and redness at the site of Bacille Calmette-Guerin (BCG) inoculation. Laboratory data were also consistent with KD (Table 1). Echocardiography did not reveal any abnormalities before treatment (left main trunk $(\mathrm{LMT})=2.4 \mathrm{~mm}, \mathrm{RCA}=2.0 \mathrm{~mm}$ ). Treatment with IVIG ( $2 \mathrm{~g} / \mathrm{kg})$, PSL ( $2 \mathrm{mg} / \mathrm{kg} /$ day), and aspirin (50 $\mathrm{mg} / \mathrm{kg} /$ day) was initiated for first-line therapy. On day 6 , his fever recurred; therefore, additional IVIG $(2 \mathrm{~g} / \mathrm{kg})$ and ulinastatin $(20,000 \mathrm{U} / \mathrm{kg})$ were administered, which effectively lowered his fever. As the RCA gradually dilated to $6.3 \mathrm{~mm}$ $(\mathrm{Z}=13.0)$ in diameter, the patient was transferred to our hospital and began treatment with warfarin $(0.1 \mathrm{mg} / \mathrm{kg} /$ day $)$ on day 9 (Fig. 1). On admission at our hospital, his temperature was $36.9^{\circ} \mathrm{C}$ and all symptoms with $\mathrm{KD}$ had disappeared. The vital signs were the following: pulse was 115 beats $/ \mathrm{min}$, respiratory rate was 25 cycles/min and his blood pressure was 95/60 $\mathrm{mm} \mathrm{Hg}$. Although his symptoms had completely disap- peared, his RCA was dilated to $7.3 \mathrm{~mm}(\mathrm{Z}=15.9)$ (Fig. 2) and his $\mathrm{C}$-reactive protein (CRP) level remained high (11.0 $\mathrm{mg} / \mathrm{dL})$. A single dose of intravenous IFX $(5 \mathrm{mg} / \mathrm{kg})$ was administered. The following day, his CRP level dramatically decreased $(4.7 \mathrm{mg} / \mathrm{dL})$ and echocardiogram revealed a giant CA with a thrombus $(4.5 \times 15.7 \mathrm{~mm})$ inside (Fig. 3). Warfarin and intravenous heparin treatment $(5,000 \mathrm{U} /$ day) successfully dissolved the thrombus. On day 16, desquamation of the fingers began and the patient's CRP level decreased to almost a normal range $(0.4 \mathrm{mg} / \mathrm{dL})$. The patient had an uneventful course during his hospital stay and was discharged on day 27. At discharge, his RCA was $13.0 \mathrm{~mm}(\mathrm{Z}=32.7)$ in diameter without a thrombus. One month after discharge, cardiac catheterization revealed that a super-giant aneurysm of $14.3 \mathrm{~mm}$ in diameter had developed in the RCA (Fig. 3). At the 4-year follow-up, the patient had not experienced a coronary event, but regression of the CA was not observed.

\section{Discussion}

We report a case of patient with IVIG-refractory KD who developed a RCA aneurysm after successful treatment with IFX. IFX is effective and well-tolerated in patients with IVIG-refractory KD; however, the timing and indication of IFX treatment should be carefully determined.

In the acute-phase treatment of $\mathrm{KD}$, it is important that IVIG begins before day 7 to prevent CA formation and should 


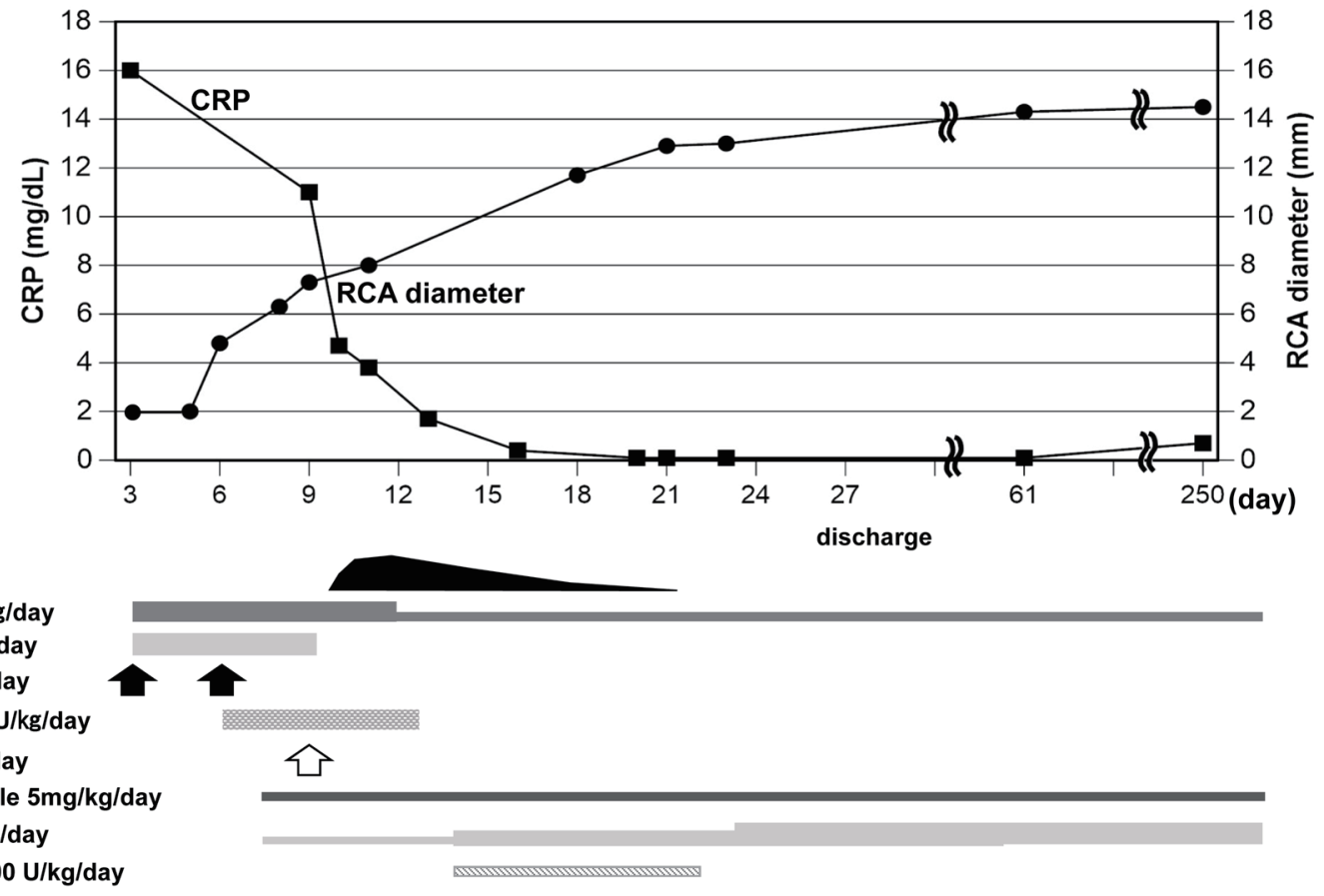

Figure 1. Clinical course after admission. Upper panel shows changes in RCA diameter and CRP levels and lower panel shows time course of applied medicine. ASA: aspirin; PSL: prednisolone; IVIG: intravenous immunoglobulin; UTI: ulinastatin; IFX: infliximab; WF: warfarin; RCA: right coronary artery; CRP: C-reactive protein.

begin before day 9 to prevent coronary artery inflammation [1] The selection and timing of second-line treatment for refractory $\mathrm{KD}$ are also essential at the early stage of treatment. Our patient required additional therapy and two rounds of IVIG, PSL, and ulinastatin, which were not effective. After the end of the second round of IVIG, his temperature returned to normal, but his CRP concentration remained high $(11.0 \mathrm{mg} / \mathrm{dL})$ and his RCA tended to dilate $(6.3 \mathrm{~mm}$ in diameter), indicating severe vasculitis. A single dose of IFX on day 9 dramatically improved laboratory data. Vascular dilatation did not stop but progressed gradually, although the timing of drug administration was reasonable. On admission at our hospital and before the administration of IFX, the patient's RCA was $7.3 \mathrm{~mm}(\mathrm{Z}=$ $15.9)$ in diameter. On day 10 it was $8.2 \mathrm{~mm}(Z=18.6)$, on day 23 it was $13.0 \mathrm{~mm}(Z=32.7)$, and ultimately his RCA dilated to $14.3 \mathrm{~mm}$ in diameter (Fig. 1).

Although the pathophysiology of CA with KD has not been fully elucidated, in 2005 Takahashi et al reported neutrophils in the coronary arterial lesions of patients who died 10 days after the onset of KD [8]. Neutrophils are involved in the damage occurring in coronary arteries in the early stage of KD. Destruction of the intima and media of coronary arteries leads to passive vasodilation, which affects blood pressure. The current model of CA formation suggests the involvement
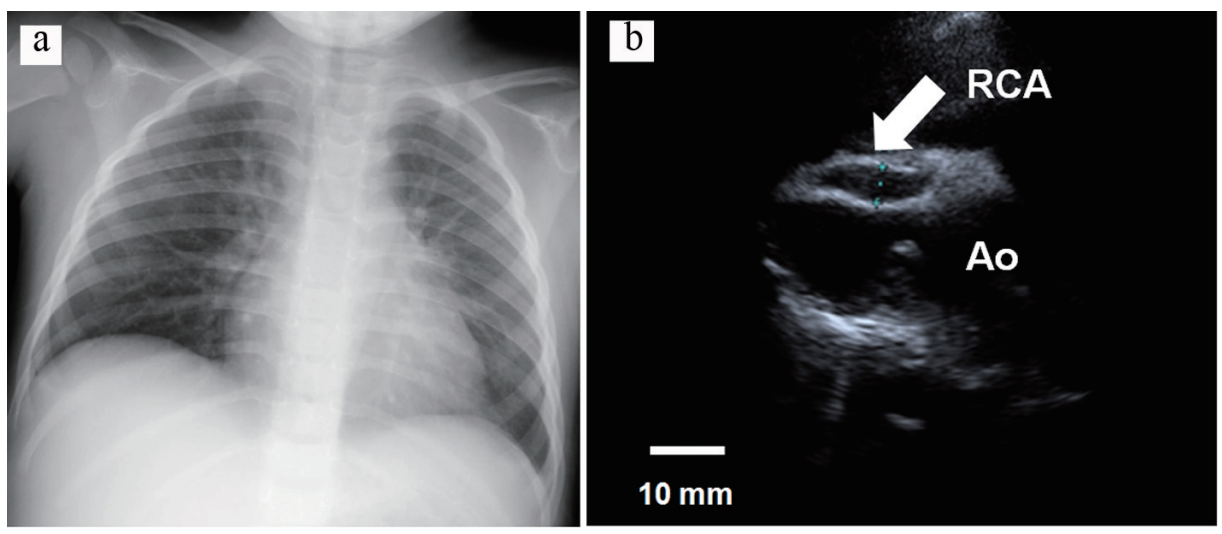

Figure 2. (a) Chest X-ray showing the CTR was $50 \%$ without cardiomegaly, and (b) transthoracic echocardiogram, showing the RCA was $7.3 \mathrm{~mm}$ in diameter and a thrombus was not detected inside the aneurysm. RCA: right coronary artery; Ao: ascending aorta; CTR: cardiothoracic ratio. 

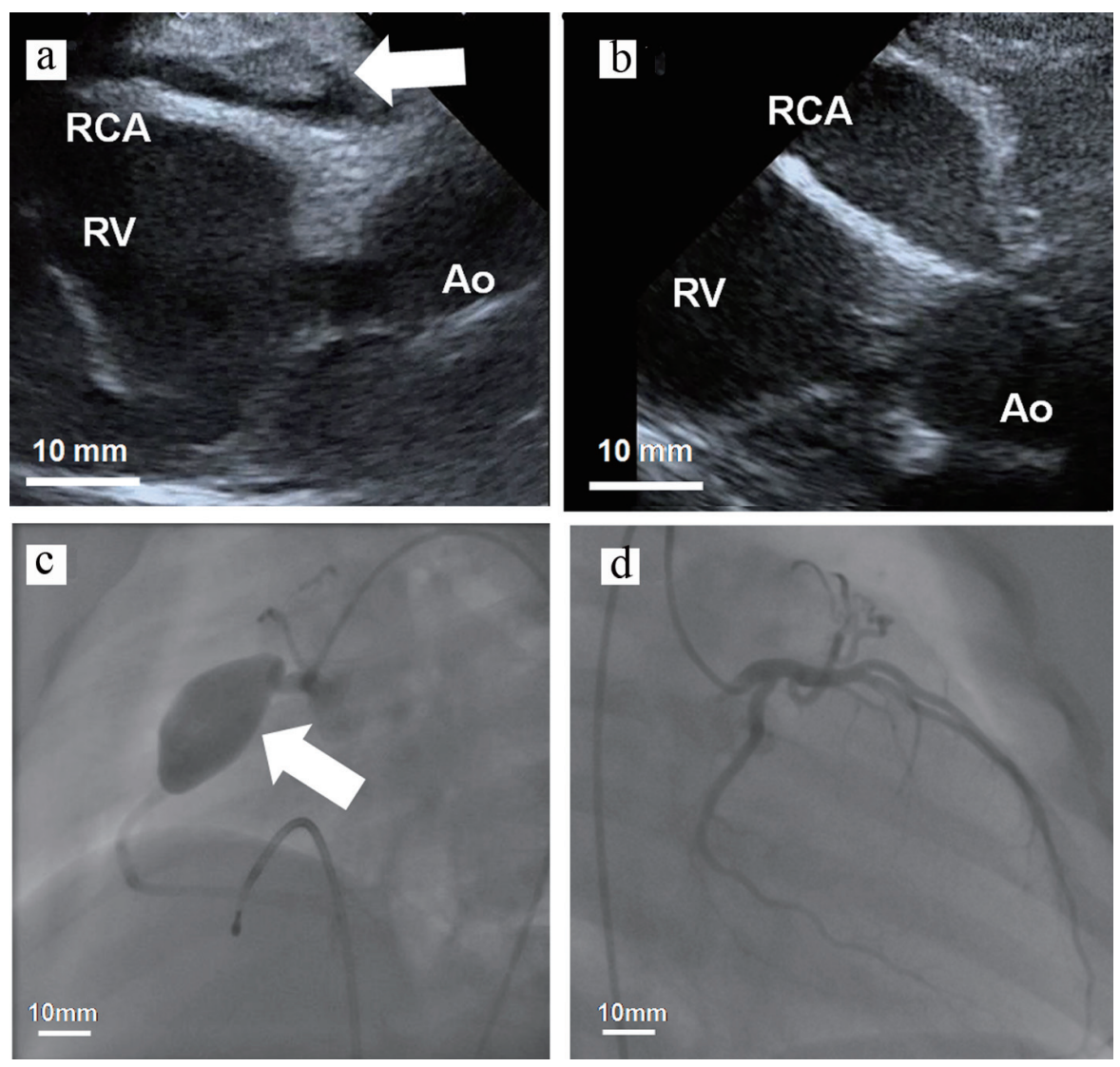

Figure 3. (a, b) Transthoracic echocardiogram and (c, d) coronary angiogram. (a) The RCA was $7.3 \mathrm{~mm}$ in diameter and a thrombus $(4.5 \times 15.7 \mathrm{~mm}$ ) was detected inside the aneurysm (day 10). (b) The size of the RCA was $14.3 \times 31.4 \mathrm{~mm}$ in diameter without a thrombus (day 61). RCA: right coronary artery; Ao: ascending aorta; RV: right ventricle.

of extracellular matrix (ECM)-degrading enzymes [1, 8-10]. The most important ECM protein in the vessel wall is elastin. The breakdown of elastin facilitates vessel wall ballooning and results in dilatation, leading to aneurysm formation.

TNF- $\alpha$ and transforming growth factor beta (TGF- $\beta$ ) are two important key regulators of elastolytic matrix metalloproteinases (MMPs), namely, the pathogenesis of CA with KD. MMP expression is induced by TNF- $\alpha$, whereas TGF- $\beta$ suppresses elastin degradation by inhibiting plasmin-mediated MMP9 activation [9]. IFX is a powerful anti-inflammatory agent. Maury et al first showed that elevated levels of TNF are characteristic of KD [10] and Lin et al showed that the serum levels of TNF- $\alpha$ were significantly higher in patients with CA than in those without CA [11]. These results suggest that the levels of circulating TNF- $\alpha$ are increased in acute KD with $\mathrm{CA}$ and support the hypothesis that this cytokine may be involved in the pathogenesis of vascular injury in KD. Burns et al first reported that IFX, a chimeric monoclonal antibody that specifically binds to TNF- $\alpha$, was effective at treating patients with refractory KD [3]. In 2018, Masuda et al used a nationwide survey of 274 Japanese institutions to analyze 434 patients administered IFX [7]. Of these patients, 363

Table 2. Summary of Previous Reports on IFX Treatment in Kawasaki Disease

\begin{tabular}{|c|c|c|c|c|c|c|}
\hline Author (reference) & $\begin{array}{l}\text { Number of } \\
\text { patients }\end{array}$ & $\operatorname{Age}(M / F)$ & $\begin{array}{l}\text { Effective } \\
\text { rate }(\%)\end{array}$ & $\begin{array}{l}\text { Illness day at } \\
\text { treatment }\end{array}$ & $\begin{array}{l}\text { Developed CA } \\
\text { after IFX (\%) }\end{array}$ & Study design \\
\hline Burns et al [3] & 16 & $2.7(0.1-13.1) 11 / 5$ & 81 & $19(8-53)$ & $0(0 / 5)$ & Retrospective \\
\hline Mori et al [5] & 20 & $4.6(1.9-10.5) 10 / 10$ & 90 & - & $5(1 / 20)$ & Open-label \\
\hline Sonoda et al [6] & 76 & $3.4(1-10) 51 / 25$ & 92 & $8.5(6-11)$ & $12(9 / 76)$ & Retrospective \\
\hline
\end{tabular}

IFX: infliximab; CA: coronary aneurysm. 
$(83.6 \%)$ became afebrile within 2 days, and the white blood cell count and serum CRP levels significantly decreased. In patients without CA before IFX, 31 patients (10.3\%) newly developed CA after IFX, and 32 patients (24.2\%) with CA before IFX exhibited increased CA severity. As a result, CA developed in one-third of patients who received IFX and a giant $\mathrm{CA}$ occurred as a complication in $5 \%$ of patients. Before IFX, nine patients had a giant $\mathrm{CA}$ and 21 had a giant $\mathrm{CA}$ after IFX (Table 2) [3-7]. The authors did not reveal the precise background of each patient or the long-term clinical course of giant CA. However, their findings suggest that IFX following IVIG resistance may have a beneficial effect on terminating the inflammatory process in acute KD patients. However, IFX may have adverse effects in patients with KD (Table 2). In our series, IFX was administered to 11 patients, with only the case described herein developing giant aneurysms (unpublished data). No patient had a CA before the administration of IFX except for this case. TGF- $\beta$ regulates T cell activities and cardiovascular remodeling. In KD, TGF- $\beta$ induces neoangiogenesis, fibrosis, and calcification by promoting the generation of myofibroblasts and inducing their transformation, as well as scarring in arteries [12]. Shimizu et al reported that genetic variants in TGFB2 and TGFBR2 are associated with KD susceptibility, coronary artery aneurysm formation, and immunoglobulin treatment response in different cohorts [13]. Additionally, their pathway analysis confirmed the importance of the TGF- $\beta$ pathway in KD pathogenesis. These data suggest that TGF signaling protects against the dilation of coronary arteries and that its dysregulation is a risk factor of CA. In severely damaged coronary arteries, few scaffolds migrate into myofibroblasts and transform into fibrosis. Millar et al reported that the use of corticosteroids in the acute phase of KD in patients with evolving coronary artery aneurysms may be associated with worsening involvement and impaired vascular remodeling [14]. Thus, IFX may also have adverse effects on dilating the coronary artery.

\section{Conclusions}

IFX is effective and safe for refractory $\mathrm{KD}$, especially for patients without CA before IFX. However, for patients with CA before IFX administration, the timing and indication of IFX treatment should be carefully determined.

\section{Acknowledgments}

We thank Dr. Eichiro Kawai, pediatrician in Tohoku University Hospital, and Dr. Kengo Kawano, pediatrician in Tohoku University Hospital, for excellent contribution to this case report.

\section{Financial Disclosure}

No honorarium or grant or other form of payment was given to anyone to produce the manuscript.

\section{Conflict of Interest}

The authors have no conflict of interest to disclose.

\section{Informed Consent}

Written informed consent for publication was obtained.

\section{Author Contributions}

SE wrote the manuscript; MK reviewed the manuscript critically and supervised it; HY and CO were involved in diagnosis and treatment; RO and SK were involved in treatment and were responsible for coordination of the work and the final version of the article; All authors have read and approved the final manuscript.

\section{Abbreviations}

CA: coronary aneurysm; IFX: infliximab; IVIG: intravenous immunoglobulin; KD: Kawasaki disease; MMP: matrix metalloproteinase; PSL: prednisolone; RCA: right coronary artery; TGF- $\beta$ : transforming growth factor beta; TNF- $\alpha$ : tumor necrosis factor-alpha; BCG: Bacille Calmette-Guerin; LMT: left main trunk; CRP: C-reactive protein; ECM: extracellular matrix

\section{References}

1. McCrindle BW, Rowley AH, Newburger JW, Burns JC, Bolger AF, Gewitz M, Baker AL, et al. Diagnosis, treatment, and long-term management of kawasaki disease: a scientific statement for health professionals from the American Heart Association. Circulation. 2017;135(17):e927e999.

2. Nakamura Y, Yashiro M, Uehara R, Sadakane A, Tsuboi S, Aoyama Y, Kotani K, et al. Epidemiologic features of Kawasaki disease in Japan: results of the 2009-2010 nationwide survey. J Epidemiol. 2012;22(3):216-221.

3. Burns JC, Mason WH, Hauger SB, Janai H, Bastian JF, Wohrley JD, Balfour I, et al. Infliximab treatment for refractory Kawasaki syndrome. J Pediatr. 2005;146(5):662667.

4. Song MS, Lee SB, Sohn S, Oh JH, Yoon KL, Han JW, Kim $\mathrm{CH}$. Infliximab treatment for refractory kawasaki disease in korean children. Korean Circ J. 2010;40(7):334-338.

5. Mori M, Imagawa T, Hara R, Kikuchi M, Hara T, Nozawa T, Miyamae T, et al. Efficacy and limitation of infliximab treatment for children with Kawasaki disease intractable to intravenous immunoglobulin therapy: report of an open-label case series. J Rheumatol. 2012;39(4):864867.

6. Sonoda K, Mori M, Hokosaki T, Yokota S. Infliximab plus plasma exchange rescue therapy in Kawasaki dis- 
ease. J Pediatr. 2014;164(5):1128-1132 e1121.

7. Masuda H, Kobayashi T, Hachiya A, Nakashima Y, Shimizu H, Nozawa T, Ogihara Y, et al. Infliximab for the Treatment of Refractory Kawasaki Disease: A Nationwide Survey in Japan. J Pediatr. 2018;195:115-120 e113.

8. Takahashi K, Oharaseki T, Naoe S, Wakayama M, Yokouchi Y. Neutrophilic involvement in the damage to coronary arteries in acute stage of Kawasaki disease. Pediatr Int. 2005;47(3):305-310.

9. Senzaki H. The pathophysiology of coronary artery aneurysms in Kawasaki disease: role of matrix metalloproteinases. Arch Dis Child. 2006;91(10):847-851.

10. Maury CP, Salo E, Pelkonen P. Elevated circulating tumor necrosis factor-alpha in patients with Kawasaki disease. J Lab Clin Med. 1989;113(5):651-654.
11. Lin CY, Lin CC, Hwang B, Chiang BN. Cytokines predict coronary aneurysm formation in Kawasaki disease patients. Eur J Pediatr. 1993;152(4):309-312.

12. Ruiz-Ortega M, Rodriguez-Vita J, Sanchez-Lopez E, Carvajal G, Egido J. TGF-beta signaling in vascular fibrosis. Cardiovasc Res. 2007;74(2):196-206.

13. Shimizu C, Jain S, Davila S, Hibberd ML, Lin KO, Molkara D, Frazer JR, et al. Transforming growth factor-beta signaling pathway in patients with Kawasaki disease. Circ Cardiovasc Genet. 2011;4(1):16-25.

14. Millar K, Manlhiot C, Yeung RS, Somji Z, McCrindle BW. Corticosteroid administration for patients with coronary artery aneurysms after Kawasaki disease may be associated with impaired regression. Int J Cardiol. 2012;154(1):9-13. 